\title{
Quercetin elicits proapoptotic effect through downregulation of antiapoptotic and upregulation of proapoptotic genes in human myeloid leukemia KG-1 cells
}

\author{
Mahmoud Vahidi ${ }^{1)}$, Ali Noroozi-Aghideh ${ }^{2)}$, Atefeh Entezari ${ }^{3)}$, Majid Zamani ${ }^{4)}$, Yoda Yaghoubi ${ }^{5)}$, \\ Ali Hassanzadeh ${ }^{6}$, Adel Naimi ${ }^{7,8)}$

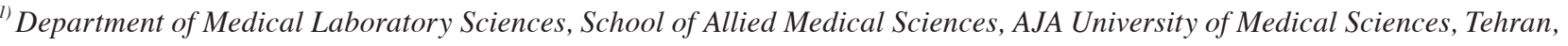 \\ Iran \\ ${ }^{2)}$ Department of Hematology, Faculty of Paramedicine, AJA University of Medical Sciences, Tehran, Iran \\ ${ }^{3)}$ Department of Medical Genetics, Faculty of Medicine, Tabriz University of Medical Sciences, Tabriz, Iran \\ 4) Department of Medical Laboratory Sciences, Faculty of Allied Medicine, Gonabad University of Medical Sciences, Gonabad, \\ Iran \\ ${ }^{5)}$ Department of Medical Laboratory Sciences, Faculty of Paramedical, Kurdistan University of Medical Sciences, Sanandaj, \\ Iran \\ ${ }^{6)}$ Department of Tissue Engineering and Applied Cell Sciences, School of Advanced Technologies in Medicine, Tehran University \\ of Medical Sciences, Tehran, Iran \\ ${ }^{7)}$ Department of Medical Laboratory Sciences, Faculty of Paramedicine, AJA University of Medical Sciences, Tehran, Iran \\ ${ }^{8)}$ Cellular and Molecular Research Center, Sabzevar University of Medical Sciences, Sabzevar, Iran
}

\begin{abstract}
Tumor necrosis factor-related apoptosis-inducing ligand (TRAIL) is a member of the tumor necrosis factor superfamily, triggering apoptosis in malignant cell without affecting healthy cells. However, varied types of the cancerous cells (e.g., leukemia) show resistance to TRAIL-induced apoptosis (TIA). Herein, we evaluated the effect of quercetin (QUE) in combination with TRAIL to defeat the resistance of the human acute myeloid leukemia KG-1 cell lines against TIA. Briefly, we treated the KG-1 cells with TRAIL $(50$ and $250 \mathrm{ng} / \mathrm{ml})$ and QUE $(100 \mu \mathrm{M})$ alone and in combination together. Then, the expression levels of the pro-apoptotic proteins, including caspase 3,8, and 9 along with the anti-apoptotic proteins such as survivin and Mcl-1 expressions levels were evaluated by real-time PCR in KG-1 cells 12, 14 and 48 hours upon exposure with TRAIL and QUE $(100 \mu \mathrm{M})$. Based on results, the combination of TRAIL and QUE augmented the expression levels of the caspase 3,8, and 9 compared with the cells treated with TRAIL and QUE alone. Conversely, expression rates of the survivin and Mcl-1 were strongly diminished in cells treated with TRAIL plus QUE in comparison to KG-1 cells that treated with TRAIL and QUE alone. Considering finding, QUE can not only improve caspase 3, 8, and 9 when used plus TRAIL, it also made a positive effect on their activation due to the inhibitory impacts on anti-apoptotic protein Mcl-1 and survivin expressions in KG-1 cells, possibly leading to the desired therapeutic outcome in leukemic cells.
\end{abstract}

Keywords: acute myeloid leukemia, apoptosis, quercetin, TNF-related apoptosis-inducing ligand (TRAIL), KG-1 cell line

(Received April 1, 2021; Accepted May 10, 2021)

\section{INTRODUCTION}

Cancer is the abnormal growth of cells in the body and the second leading cause of death in the world. Leukemia is a group of hematologic cancers which initiates the disease in hematopoietic system ${ }^{1,2)}$. Despite the high mortality rate of leukemia, no cure has been yet

Mahmoud Vahidi and Ali Noroozi-Aghideh contributed equally to this work

Corresponding author: Adel Naimi, Department of Medical Laboratory Sciences, Faculty of Paramedicine, AJA University of Medical Sciences, Tehran, Iran. Tel: +98 5144018369, E-mail: naeimia@medsab.ac.ir found that can effectively overcome the diseases ${ }^{3)}$. The primary goal of cancer treatment researches during the past decades has been to develop the strategies to eliminate the cancer cells without affecting normal cells ${ }^{4)}$. The selective induction of type I programmed cell death or apoptosis in the malignant cells can be considered as one of the strategies which successfully helps us to eliminate the cancer cells. The apoptosis program can be triggered by either of the intrinsic or extrinsic pathways ${ }^{5)}$. The ligation of members of the tumor necrosis factor (TNF) superfamily to its receptor on the cell surface is enough to activate the extrinsic cell death pathway ${ }^{6,7)}$. The TNF-related apoptosis-inducing ligand (TRAIL), as a member of the TNF superfamily, has been reported 
that can induce the apoptosis process without affecting the healthy cells in a variety of cancer cell lines ${ }^{8)}$. Despite the destructive effects of TRAIL on cancer cells, several studies have revealed that some cancers can acquire resistance to TRAIL-induced apoptosis (TIA) ${ }^{9,10)}$. Hence, there are still a lot of barriers to use TRAIL as an anticancer agent. In this regard, more recent studies have confirmed that the combine treatment of TRAIL with the sensitizer agents, such as chemotherapy agents, natural compounds or radiation can be considered as a solution to overcome this problem ${ }^{11)}$. Besides, there is a large volume of published studies describing the pharmacological effects of flavonoids such as the antioxidant activities and the selective antiproliferative roles in cancer cells. Also, several recent findings have shown that quercetin (QUE) is a dietary flavonoid which can trigger the cell death, mainly by initiating the apoptosis process in leukemia cells without affecting the normal cells $^{12,13)}$. Based on investigation, QUE could suppress human HL-60 promyelocytic leukemia cell proliferation and promote their apoptosis percentages by targeting protein kinase $\mathrm{C}(\mathrm{PKC})$ and tyrosine protein kinase (TPK) pathways ${ }^{14)}$. Another study indicated that QUE ameliorated mitochondrial function by stimulating the proteasomal degradation of Mcl-1 (myeloid cell leukemia 1), a pro-survival member of the Bcl-2 family and by suppression of the expression of survivin, a member of the inhibitor of apoptosis (IAP) family, in non-Hodgkin's lymphoma ${ }^{15)}$. Furthermore, it has been evidenced that QUE may be valuable therapeutic approach to treat human leukemia U937 cells by triggering apoptosis in leukemia versus normal hematopoietic cells by a procedure, encompassing Mcl-1 down-regulation, supporting Bax activation and mitochondrial translocation, and thereby culminating apoptosis ${ }^{16)}$. Besides, reports revealed that QUE down-regulated Mcl-1 by the suppression of PI3K/ Akt signaling pathway, promoting Mcl-1 instability in leukemia cells, which in turn, led to the apoptosis ${ }^{17)}$.

In the present study, we have co-treated the human myeloid leukemia KG-1 cell lines with TRAIL and QUE. Then, we investigated whether this co-treatment can change the messenger RNA (mRNA) expression levels of Caspase-3, 8, and 9 and the apoptosis inhibitors including survivin and Mcl-1 or not.

\section{MATERIALS AND METHODS}

\section{Cell culture}

Human myeloid leukemia KG-1 cells were purchased from General Cell Collection Bank of Iran (Pasteur Institute, Tehran, Iran). The KG-1 cell line maintained in exponential growth in RPMI-1640 (Gibco, Paisley, UK) supplemented with $10 \%$ (v/v) fetal bovine serum (FBS; Sigma-Aldrich, Gillingham, UK), $50 \mu \mathrm{g} / \mathrm{ml}$ of streptomycin (Sigma-Aldrich), and 50 units $/ \mathrm{ml}$ of penicillin
(Sigma-Aldrich) at $37^{\circ} \mathrm{C}$ in a humidified atmosphere containing $5 \% \mathrm{CO}_{2}$.

\section{Reagents}

A 100 mM stock solution of QUE (>99\% pure; SigmaAldrich) was dissolved in dimethyl sulfoxide (DMSO), stored at $-20^{\circ} \mathrm{C}$, and then diluted as needed in RPMI1640 to its final concentration in each culture dish. Also, a $0.5 \mathrm{mg} / \mathrm{ml}$ stock solution of the recombinant human Apo2L/TRAIL (Merck, Darmstadt, Germany), was prepared in water. RPMI-1640 medium, FBS, penicillin, streptomycin, and phosphate buffered saline were purchased from Gibco (Carlsbad, USA). DMSO was obtained from Sigma-Aldrich.

RNA extraction and Quantitative real-time polymerase chain reaction

The KG-1 cells treated with TRAIL (50 and $250 \mathrm{ng} / \mathrm{ml})$ and QUE $(100 \mu \mathrm{M})$ alone and in combination at the 12, 24, and 48 hours. Then, the cells harvested for RNA extraction using Trizol Reagent (Invitrogen, Milan, Italy) following the manufacturer's instructions. The quality and concentration of the RNA samples was assessed by NanoDrop 2000c UV-Vis spectrophotometer (Thermo Fisher Scientific, Waltham, USA). The recommended RNA quality parameters for Real-time PCR analysis were as follows: UV spectroscopy A260/ A280 ratio of 1.8-2.0. 500 nanograms of the extracted RNA were reverse-transcribed into cDNA using, PrimeScript ${ }^{\mathrm{TM}}$ reagent Kit (Takara Bio, Kusatsu, Japan). Real-time PCR analysis was done using the using the RealQ Plus $2 x$ Master Mix Green (Ampliqon, Herlev, Denmark) following the manufacturer's protocol. Quantification and the analysis of melting curves was performed using the real-time PCR system (Rotor-Gene 6000; Corbett Life Science, Sydney, Australia). Also, we used the primerblast software on NCBI website (http://www. nchi.nlm. nih.gov) to Basic Local Alignment Search Tool (BLAST) our all primer sequences before the experiment. Betaactin (ACTB) was used as an internal control. The primers used in this study and target sequences data listed in Table 1 .

\section{Statistical analysis}

Statistical analyses were performed using the GraphPad Prism version 8.3.0 for Windows (GraphPad Software Inc, San Diego, USA). Gene expression data was analyzed for any statistically significant using a twoway analysis of variance with the post hoc Dunnett and Tukey multiple comparison tests. Data were presented as the mean \pm standard error of the mean (SEM) and were representative for three separate experiments. $\mathrm{P}$ values $<0.05$ were considered to be statistically significant. 
Table 1. Primer Sequences, All of the sequences of the primers are presented in 5 'to 3' orientation.

\begin{tabular}{c|c|c}
\hline Gene & Forward Primers $\left(5^{\prime}-3^{\prime}\right)$ & Reverse Primers $\left(5^{\prime}{ }^{\prime}-3^{\prime}\right)$ \\
\hline Caspase 3 & TGTTTGTGTGCTTCTGAGCC & CACGCCATGTCATCATCAAC \\
\hline Caspase 8 & GATCAAGCCCCACGATGAC & CCTGTCCATCAGTGCCATAG \\
\hline Caspase 9 & CATTTCATGGTGGAGGTGAAG & GGGAACTGCAGGTGGCTG \\
\hline Survivin & TTCTCAAGGACCACCGCATC & GCCAAGTCTGGCTCGTTCTC \\
\hline Mcl-1 & AGAAAGCTGCATCGAACCAT & CCAGCTCCTACTCCAGCAAC \\
\hline ACTB & TGAAGATCAAGATCATTGCTCCC & AGTCATAGTCCGCCTAGAAGC \\
\hline
\end{tabular}

\section{RESULTS}

QUE increased the expression levels of Caspase 3 gene in the human myeloid leukemia KG-1 cells (at transcriptional level)

To evaluate the QUE effect on caspase 3 expression in KG-1 cells, which constitute an in vitro model of human acute myeloid leukemia, we employed the quantitative real-time polymerase chain reaction analysis to compare the QUE treated and untreated cells. Based on the results, the caspase 3 expression did not change significantly in cells treated with TRAIL alone during 12, 14 and 48 hours of treatment compared with the control group ( $\mathrm{P}$ $<0.05)$; however, its expression levels were significantly augmented in KG-1 cells treated with QUE $(100 \mu \mathrm{M})$ alone and in combination with TRAIL (50 and $250 \mathrm{nM}$ ) during 12, 14 and 48 hours of treatment. Moreover, the expression levels of caspase 3 were more elevated in cells treated with QUE in combination with TRAIL during 12 and 24 hours than 48 hours of exposure. The results are shown in Fig. 1.

QUE increased the expression levels of caspase 8, and 9 genes in the human myeloid leukemia KG-1 cells (at transcriptional level)

In this regard, to investigate the effect of QUE on caspase 8, and 9 as the initiator caspases in apoptosis, we evaluated their mRNA expression levels after treating the KG-1 cells with TRAIL and QUE during the indicated times. Although the caspase 8, and 9 expression did not modify significantly in cells treated with TRAIL alone during 12, 14 and 48 hours of treatment, their expression levels were significantly promoted in KG-1 cells treated with QUE $(100 \mu \mathrm{M})$ alone and in combination with TRAIL (50 and $250 \mathrm{nM}$ ) during 12, 14 and 48 hours of

\section{Caspase 3}

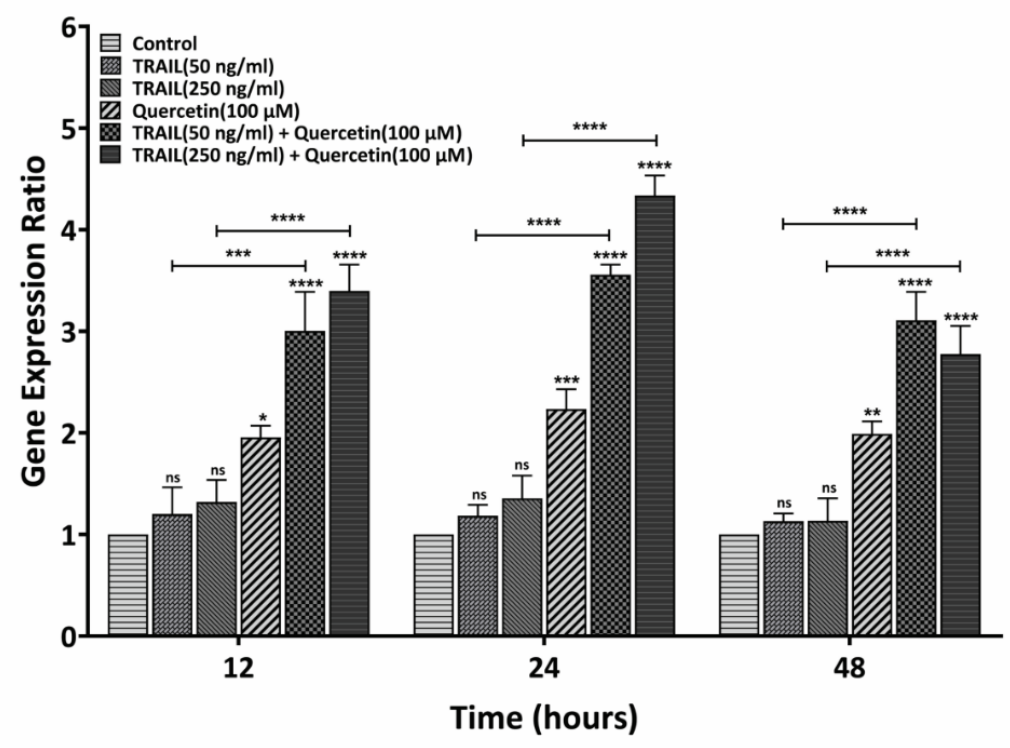

Fig. 1. Real-Time PCR data for caspase 3 expression in KG-1 cells treated with TRAIL (50 and $250 \mathrm{nM})$ and QUE (100 $\mu \mathrm{M})$ alone and in combination within 12, 14 and 48 hours of treatment. Data are representative of three independent experiments and values are expressed in mean \pm SEM. ACTB was selected as internal control. One-Way ANOVA was used to evaluate the observed statistical differences. $P$ values $<0.05$ were considered statistically significant. 
A

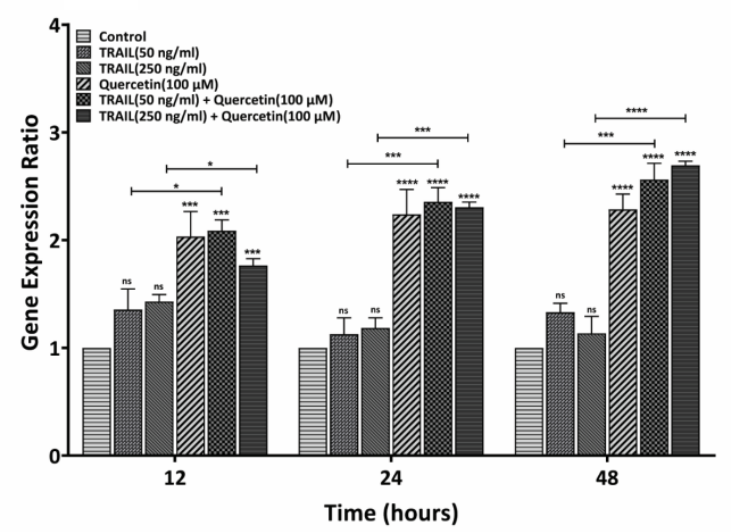

B

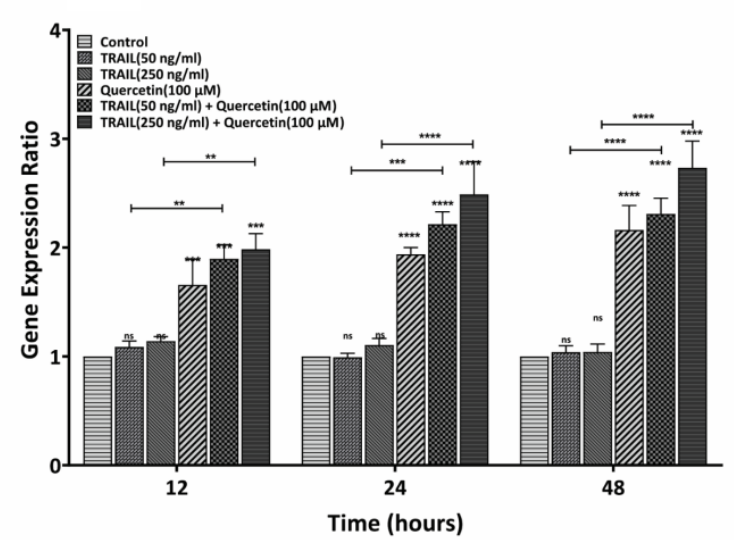

Fig. 2. Real-Time PCR data for caspase 8 (A) and caspase 9 (B) expression in KG-1 cells treated with TRAIL (50 and $250 \mathrm{nM}$ ) and QUE $(100 \mu \mathrm{M})$ alone and in combination within 12, 14 and 48 hours of treatment. Data are representative of three independent experiments and values are expressed in mean \pm SEM. ACTB was selected as internal control. One-Way ANOVA was used to evaluate the observed statistical differences. P values $<0.05$ were considered statistically significant.

A

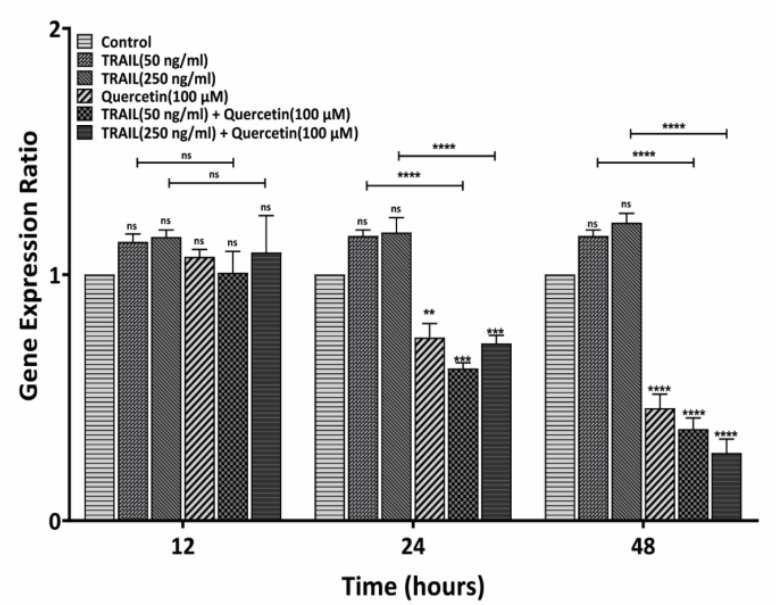

B

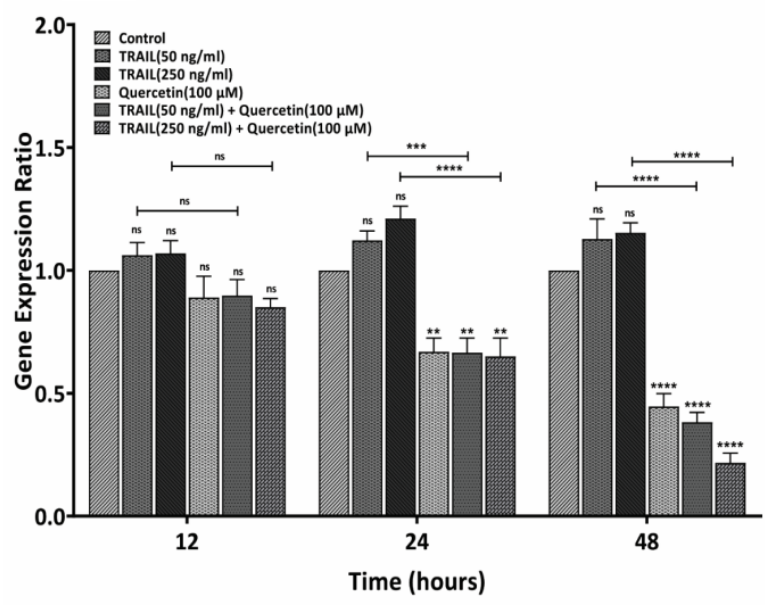

Fig. 3. Real-Time PCR data for Mcl-1 (A) and survivin (B) expression in KG-1 cells treated with TRAIL (50 and $250 \mathrm{nM}$ ) and QUE $(100 \mu \mathrm{M})$ alone and in combination within 12,14 and 48 hours of treatment. Data are representative of three independent experiments and values are expressed in mean \pm SEM. ACTB was selected as internal control. One-Way ANOVA was used to evaluate the observed statistical differences. $P$ values $<0.05$ were considered statistically significant.

treatment compared with the control group $(\mathrm{P}<0.05)$. Furthermore, the expression levels of the caspase 8 and 9 were higher in KG-1 cells treated with QUE in combination with TRAIL within 48 hours than the 12 and 24 hours of treatment. The real-time PCR results are shown in Fig. 2.

QUE decreased the expression of apoptosis inhibitor genes in the acute myeloid KG-1 cells (at transcriptional level)

Finally, in this part, we attempted to investigate the effect of quercetin on the expression levels of Mcl-1 and survivin at transcriptional levels in KG-1 cells treated with TRAIL (50 and $250 \mathrm{nM}$ ) and QUE $(100 \mu \mathrm{M})$ alone within 12, 14 and 48 hours of exposure. Many researchers have argued that apoptosis inhibitors can act as an important factor in developing cancer in the abnormal cells. As shown in Fig. 3A, the QUE treatment of KG-1 cells has been able to significantly reduce the mRNA expression levels of Mcl-1 either in QUE alone or in combination with TRAIL-treated cells during the indicated times compared with the control group $(\mathrm{P}<0.05)$. The 
survivin gene expression changes that has been plotted in Fig. 3B clearly shows that these changes are roughly similar to the Mcl-1 gene at the transcriptional level compared with control group $(\mathrm{P}<0.05)$. Considering analysis, inhibitory effects of the QUE on Mcl-1 and survivin expression were stronger in 48 hours than 12 and 24 hours of exposure.

\section{DISCUSSION}

TRAIL, as a biological cytokine, is attracting attention as a potential therapeutic agent that selectively can trigger apoptosis in cancer cells but not in normal cells $^{18,19)}$. But the therapeutic usage of this cytokine is limited due to resistance of some cancer cells to the TRAIL induced apoptosis (TIA) ${ }^{20,21)}$. Hence, introducing new sensitizer agents can sensitize resistant cancer cells to TIA. Recent evidences suggest that QUE as a plant flavonol can be used as a sensitizing agent alongside with TRAIL in some cancer cells ${ }^{22,23)}$. In this context, Kim and his coworkers found that QUE promoted TIA by suppression of survivin expression, through ERK-MSK1mediated deacetylation of $\mathrm{H} 3$ in in human prostate cancer cell lines DU-145 and PC-3. They suggested that up regulation of caspase- $8,-9$, and -3 along with down regulation of survivin could remarkably stimulate apoptosis in treated cells with QUE plus TRAIL ${ }^{24)}$. Similarly, another study conducted by Psahoulia et al. showed that QUE plus TRAIL triggered the apoptosis intrinsic pathway, as presented by Bid cleavage and the cytochrome c release to the cytosol, accompanied by redistribution of DR4 and DR5 into lipid rafts in colorectal cancer $(\mathrm{CRC})^{25)}$. Moreover, QUE plus TRAIL augmented apoptosis in hepatocellular carcinoma (HCC) cells upon DR5 up regulation in a transcription factor $\mathrm{Sp1}$ - dependent manner in concomitant with down regulation c-FLIP, an inhibitor of caspase-8, by proteasome - exerted degrada$\operatorname{tion}^{26)}$. As well, suppression of survivin expression and activation in association with DR5 up regulation were considered as corresponding mechanisms participated TRAIL-mediated apoptosis in non-small cell lung cancer (NSCLC) cells upon exposure with QUE, according to Chen et al. reports ${ }^{27)}$. However, the mechanism of action of QUE in sensitizing leukemia cells (e.g., KG-1) to the TIA is not well understood. In our recent study we demonstrated that QUE alone or alongside with the use of TRAIL could augment the TIA in the resistant acute myeloid KG-1 cells to TIA ${ }^{28)}$. Analysis revealed that quercetin represses the expression of NF- $x \mathrm{~B}$ (p65 subunit) gene in $\mathrm{KG}-1$ cells $^{22)}$. This finding is consistent with findings of other studies that are emphasizing on the possible role of the inhibition of NF- $x \mathrm{~B}$ for the caspase-3 upregulation. The NF- $x \mathrm{~B}$ prevents apoptosis through increasing the expression of several Bcl-2 family members genes that these targets prevent apoptosis ${ }^{29)}$. It can be concluded that among the factors that have been able to increase the rate of apoptosis in KG-1 cells are NF- $x$ B targets that affect the release of cytochrome $\mathrm{c}$ from mitochondria. Additionally, the inhibitory effect of quercetin on NF$x \mathrm{~B}$ expression, leading to the increased expression of caspases including caspases 3,8 and 9, may be one of the main mechanisms in sensitizing KG-1 cells to TIA. The aim of the present work is to investigate the further anticancer effects of QUE on KG-1 cells. Accordingly, firstly, we evaluated expression levels of anti-apoptotic proteins Mcl-1 and survivin in the KG-1 cells upon exposure with TRAIL and QUE alone and in combination. It has been already been found that overexpression of anti-apoptotic proteins, in particular Mcl-1 and survivin, is one of the main reason of cancer cells resistance to TIA ${ }^{30,31)}$. Considering molecular analysis, we found that QUE stimulated an inhibitory effect on Mcl-1 and surviving expression levels when used along with TRAIL. There exist a consistency between these results and other findings in varied types of the cancer cells (e.g., leukemia). For example, Jacquemin et al. found that QUE induced TIA in resistant transformed follicular lymphoma B-cell lines through the triggering the proteasomal degradation of Mcl-1 accompanied by suppressing survivin expression, irrespective of $\mathrm{p} 53^{15)}$.

Secondly, examination of QUE and TRAIL effects on mRNA levels of the pro-apoptotic factors, such as caspase 3, 8, and 9, delivered the proof of concept that QUE can triggered TIA in KG-1 cells by amelioration of the activator and effector caspase expression. These findings are consistent with the results of previous studies exploring the effect of QUE on TIA in other tumor cells ${ }^{32)}$.

In sum, studies on the anti-leukemic potential of flavonoids are described a stimulating topics of the context of the leukemia therapy because of their lower unwanted effect and notable safety. In the present study, we found that QUE can robustly promote aspase-3, -8 and -9 expression in leukemia KG-1 cells; on the other hand, it stimulated a reduction in anti-apoptotic protein Mcl1 and survivin expression in mRNA levels, which in turn can lead to the apoptosis induction in treated cells. Accordingly, we guess that QUE can be a rational drugcandidate to progress the novel anti-leukemia therapeutics toward a spectrum of leukemia with minimum sideeffects and suitable rates of safety along with acceptable efficacy.

\section{Declaration of competing interest:}

The authors declare that they have no known competing financial interests or personal relationships that could have appeared to influence the work reported in this paper.

Acknowledgments:

This study was supported by a research grant from the AJA University of Medical Sciences, Tehran, Iran. 


\section{References}

1) Abruzzese E., Trawinska M. M., de Fabritiis P. and Baccarani M. (2016) Management of pregnant chronic myeloid leukemia patients. Expert Rev Hematol. 9: 781-791.

2) Cheung E., Perissinotti A. J., Bixby D. L., Burke P. W., Pettit K. M., Benitez L. L., Brown J., Scappaticci G. B. and Marini B. L. (2019) The leukemia strikes back: a review of pathogenesis and treatment of secondary AML. Ann Hematol. 98: 541-559.

3) Burnett A., Wetzler M. and Lowenberg B. (2011) Therapeutic advances in acute myeloid leukemia. J Clin Oncol. 29: 487-494.

4) Kaufmann S. H. and Steensma D. (2005) On the TRAIL of a new therapy for leukemia. Leukemia. 19: 2195-2202.

5) Almasan A. and Ashkenazi A. (2003) Apo2L/TRAIL: apoptosis signaling, biology, and potential for cancer therapy. Cytokine Growth Factor Rev. 14: 337-348.

6) Haralambiev L., Wien L., Gelbrich N., Kramer A., Mustea A., Burchardt M., Ekkernkamp A., Stope M. B. and Guembel D. (2019) The effects of cold atmospheric plasma on the expression of chemokines, growth factors, TNF superfamily members, interleukins, and cytokines in human osteosarcoma cells. Anticancer Res. 39: 151-157.

7) Lee W.-H., Seo D., Lim S.-G. and Suk K. (2019) Reverse signaling of tumor necrosis factor superfamily proteins in macrophages and microglia: Superfamily portrait in the neuroimmune interface. Front immunol. 10: 262.

8) Naimi A., Movassaghpour A. A., Hagh M. F., Talebi M., Entezari A., Jadidi-Niaragh F. and Solali S. (2018) TNF-related apoptosisinducing ligand (TRAIL) as the potential therapeutic target in hematological malignancies. Biomed Pharmacother. 98: 566-576.

9) Lee T. J., Lee J. T., Park J. W. and Kwon T. K. (2006) Acquired TRAIL resistance in human breast cancer cells are caused by the sustained cFLIP(L) and XIAP protein levels and ERK activation. Biochem Biophys Res Commun. 351: 1024-1030.

10) Xu J., Zhou J.-Y., Wei W.-Z. and Wu G. S. (2010) Activation of the Akt survival pathway contributes to TRAIL resistance in cancer cells. PloS one. 5: e10226.

11) Hassanzadeh A., Farshdousti Hagh M., Alivand M. R., Akbari A. A. M., Shams Asenjan K., Saraei R. and Solali S. (2018) Downregulation of intracellular anti-apoptotic proteins, particularly cFLIP by therapeutic agents; the novel view to overcome resistance to TRAIL. J Cell Physiol. 233: 6470-6485.

12) Sadhukhan P., Kundu M., Chatterjee S., Ghosh N., Manna P., Das J. and Sil P. C. (2019) Targeted delivery of quercetin via pH-responsive zinc oxide nanoparticles for breast cancer therapy.: C. Mater Sci Eng C Mater Biol Appl. 100: 129-140.

13) Riaz M. K., Zhang X., Wong K. H., Chen H., Liu Q., Chen X., Zhang G., Lu A. and Yang Z. (2019) Pulmonary delivery of transferrin receptors targeting peptide surface-functionalized liposomes augments the chemotherapeutic effect of quercetin in lung cancer therapy. Int J Nanomedicine. 14: 2879-2902.

14) Kang T.-b. and Liang N.-c. (1997) Studies on the inhibitory effects of quercetin on the growth of HL-60 leukemia cells. Biochem Pharmacol. 54: 1013-1018.

15) Jacquemin G., Granci V., Gallouet A. S., Lalaoui N., Morlé A., Iessi E., Morizot A., Garrido C., Guillaudeux T. and Micheau O. (2012) Quercetin-mediated Mcl-1 and survivin downregulation restores TRAIL-induced apoptosis in non-Hodgkin's lymphoma B cells. Haematologica. 97: 38-46.

16) Cheng S., Gao N., Zhang Z., Chen G., Budhraja A., Ke Z., Son Y.o., Wang X., Luo J. and Shi X. (2010) Quercetin induces tumorselective apoptosis through downregulation of Mcl-1 and activation of Bax. Clin Cancer Res. 16: 5679-5691.

17) Russo M., Spagnuolo C., Volpe S., Tedesco I., Bilotto S. and Russo G. L. (2013) ABT-737 resistance in B-cells isolated from chronic lymphocytic leukemia patients and leukemia cell lines is overcome by the pleiotropic kinase inhibitor quercetin through Mcl-1 downregulation. Biochem Pharmacol. 85: 927-936.
18) Hassanzadeh A., Naimi A., Hagh M. F., Saraei R., Marofi F. and Solali S. (2019) Kaempferol improves TRAIL-mediated apoptosis in leukemia MOLT-4 cells by the inhibition of anti-apoptotic proteins and promotion of death receptors expression. Anicancer Agents Med Chem. 19: 1835-1845.

19) Naimi A., Safaei S., Entezari A., Solali S. and Hassanzadeh A. (2020) Knockdown of Enhancer of Zeste Homolog 2 Affects mRNA Expression of Genes Involved in the Induction of Resistance to Apoptosis in MOLT-4 Cells. Anticancer Agents Med Chem. 20: 571-579.

20) Sharma A. and Almasan A. (2018) Autophagy as a mechanism of Apo2L/TRAIL resistance. Cancer Biol Ther. 19: 755-762.

21) Zhu Z.-C., Liu J.-W., Yang C., Li M.-J., Wu R.-J. and Xiong Z.-Q. (2019) Targeting KPNB1 overcomes TRAIL resistance by regulating DR5, Mcl-1 and FLIP in glioblastoma cells. Cell Death Dis. 10: 118.

22) Kim J. H., Kim M. J., Choi K.-C. and Son J. (2016) Quercetin sensitizes pancreatic cancer cells to TRAIL-induced apoptosis through JNK-mediated cFLIP turnover. Int J Biochem Cell Bio. 78: $327-734$.

23) Hassanzadeh A., Hosseinzadeh E., Rezapour S., Vahedi G., Haghnavaz N. and Marofi F. (2019) Quercetin promotes cell cycle arrest and apoptosis and attenuates the proliferation of human chronic myeloid leukemia cell line-K562 through interaction with HSPs (70 and 90), MAT2A and FOXM1. Anticancer Agents Med Chem. 19: 1523-1534.

24) Kim Y.-H., Lee D.-H., Jeong J.-H., Guo Z. S. and Lee Y. J. (2008) Quercetin augments TRAIL-induced apoptotic death: Involvement of the ERK signal transduction pathway. Biochem Pharmacol. 75: 1946-1958.

25) Psahoulia F. H., Drosopoulos K. G., Doubravska L., Andera L. and Pintzas A. (2007) Quercetin enhances TRAIL-mediated apoptosis in colon cancer cells by inducing the accumulation of death receptors in lipid rafts. Mol Cancer Ther. 6: 2591-2599.

26) Kim J. Y., Kim E. H., Park S. S., Lim J. H., Kwon T. K. and Choi K. S. (2008) Quercetin sensitizes human hepatoma cells to TRAILinduced apoptosis via Sp1-mediated DR5 up-regulation and proteasome-mediated c-FLIPS down-regulation. J Cell Biochem. 105: 1386-1398.

27) Chen W., Wang X., Zhuang J., Zhang L. and Lin Y. (2007) Induction of death receptor 5 and suppression of survivin contribute to sensitization of TRAIL-induced cytotoxicity by quercetin in non-small cell lung cancer cells. Carcinogenesis. 28: 2114-2121.

28) Naimi A., Entezari A., Hagh M. F., Hassanzadeh A., Saraei R. and Solali S. (2019) Quercetin sensitizes human myeloid leukemia KG-1 cells against TRAIL-induced apoptosis. J Cell Physiol. 234: 13233-13241.

29) Kiekow C. J., Figueiró F., Dietrich F., Vechia L. D., Pires E. N. S., Jandrey E. H. F., Gnoatto S. C. B., Salbego C. G., Battastini A. M. O. and Gosmann G. (2016) Quercetin derivative induces cell death in glioma cells by modulating NF- $\varkappa \mathrm{B}$ nuclear translocation and caspase-3 activation. Eur J Pharm Sci. 84: 116-122.

30) Woo S. M., Min K.-j., Seo B. R. and Kwon T. K. (2016) YM155 sensitizes TRAIL-induced apoptosis through cathepsin S-dependent down-regulation of Mcl-1 and NF- $\varkappa$ B-mediated down-regulation of c-FLIP expression in human renal carcinoma Caki cells. Oncotarget. 7: 61520 .

31) Han M. A., Lee D. H., Woo S. M., Seo B. R., Min K.-j., Kim S., Park J.-W., Kim S. H., Choi Y. H. and Kwon T. K. (2016) Galangin sensitizes TRAIL-induced apoptosis through down-regulation of anti-apoptotic proteins in renal carcinoma Caki cells. Sci Rep. 6: $1-10$.

32) Brisdelli F., Coccia C., Cinque B., Cifone M. G. and Bozzi A. (2007) Induction of apoptosis by quercetin: different response of human chronic myeloid (K562) and acute lymphoblastic (HSB-2) leukemia cells. Mol Cell Biochem. 296: 137-149. 